\title{
Factors Influencing Private Practitioners to Report Tuberculosis Cases
}

Anneke Rosma ${ }^{1,2}$, Chiho Yamazaki ${ }^{1}$, Satomi Kameo ${ }^{1}$, Dewi MD. Herawati² ${ }^{2}$ Rovina Ruslami², Ardini Raksanagara $^{2}$, Hiroshi Koyama ${ }^{1}$

${ }^{1}$ Department of Public Health, Gunma University, Maebashi, Gunma, Japan.

${ }^{2}$ Faculty of Medicine, Universitas Padjadjaran, Jatinangor, West Java, Indonesia.

\begin{abstract}
This study aimed to investigate the present condition of tuberculosis (TB) cases recordingreporting in the Babakan Surabaya primary health center (PHC) and evaluate several factors that might influence private practitioners' (PP) behavior regarding the reporting of tuberculosis cases to PHC. Face-to-face interviews with PP and the head of PHC were performed. Data were coded, categorized, and analyzed statistically by non-parametric statistics (Fischer Exact Test) using EZR. We found that there were four factors affecting TB cases reporting by PP to PHC, including self-awareness, ignorance, lack of time, and poor implementation of recording-reporting at the PHC. The level of PP self-awareness was significantly associated with the reporting of TB cases by PP $(\mathrm{p}<0.05)$. Private sector involvement, improvement in the recording-reporting of treatment follow-up, and the use of electronic-based reporting were considered important by participants to construct a well-established recording-reporting system for TB cases. Therefore, good communication and collaboration between PHC and PP are needed.
\end{abstract}

Keywords: recording-reporting, tuberculosis, self-awareness, communication and collaboration, practitioners.

\section{Introduction}

Tuberculosis (TB) is an infectious disease caused by Mycobacterium tuberculosis. TB typically affects the lungs, but it can also affect other parts of the body, such as brain, kidneys, and the spine. ${ }^{1,2}$ The United Nations has defined one of the targets of Millennium Development Goals, including the reverse the incidence of TB by $2015 .^{3}$ Nevertheless, TB remains a major global health problem. In 2012, 8.6 million people developed TB and 1.3 million died from this disease. ${ }^{4,5}$ Without treatment, TB mortality rates are high. In the studies regarding the natural history of TB among sputum smear-positive cases, approximately $70 \%$ died within 10 years. $^{6}$

Indonesia has a high prevalence of $\mathrm{TB}$ and is one of the 22 countries with the highest TB burdens in the world. ${ }^{7}$ Direct Observed Treatment Shortcourse (DOTS) strategy has been introduced to reduce TB burden. The DOTS strategy comprises five elements, including political commitment

Corresponding author: Anneke Rosma, Department of Public Health, Gunma University, Maebashi, Gunma, Japan. Email : rosma00777@gmail.com 
with increased and sustained financing, cases detection through quality-assured bacteriology/sputum-smear microscopic examination, standardized treatment with supervision and patient support, an effective drug supply and management system and monitoring, evaluation system, and impact measurement through a recording and reporting system..$^{8-9}$ Indonesia has adopted the DOTS strategy since 1995. Moreover, it has been implemented by all primary health centers (PHC) in Indonesia since 2000.10 DOTS activities in Indonesia are based on the International Standards of TB Care (ISTC). ISTC provides a set of widely accepted standards that all practitioners (public and private) should seek to meet. It aims to maintain the health of individuals with TB, prevent disease in their families and others with whom they come in contact and protect the health of communities. Therefore, in Indonesia, private practitioners (PP) and PHC have similar activities in implementing the DOTS strategy. All health care providers should report tuberculosis cases detected to an authorized PHC. ${ }^{11,12}$

DOTS is applied differently in Japan. In particular, DOTS and the Japanese modification of DOTS differ in the case detection and contact tracing. Recording and reporting is one of the five DOTS components. Without good recording and reporting, there will be no evaluation for the improvement of TB programs and prevention of TB spreading among communities. ${ }^{13}$

According to the 2011 West Java Health Profile, Bandung, the capital city of West Java Province, has been ranked the 10th city with the highest tuberculosis prevalence. Bandung is also the most densely populated city in the West Java Province (14,505 persons per square kilometer). Population density is considered a factor that can worsen tuberculosis spread in a community. ${ }^{14,15}$

PHC as health care facilities at the grass roots level, plays a major role in the reduction of TB prevalence. Bandung has 73 primary PHC. Babakan Surabaya PHC is considered as one of the ten primary health centers with the lowest coverage of sputum smear-positive case finding in Bandung. The estimated number of sputum smear-positive cases is defined as the incidence rate in the city multiplied by the total population in the area. The estimated incidence rate in Bandung was 107 per 100,000 population. In 2012, the total population in the working area of the center was 36,088 people. The estimated number of sputum smear-positive cases was 38 . Meanwhile, the center reported 14 cases. ${ }^{16}$ This implies that the coverage of sputum smear-positive case finding in the center was only $36 \%$. Few private practitioners regularly reported cases to the PHC. This situation can be summarized by saying that many tuberculosis cases are not reported. This will lead to the absence of contact tracing and patients' treatment follow-up. Furthermore, it will increase the spread of tuberculosis and drug resistance.

The aim of this study was to investigate several factors that might influence PP behavior regarding the reporting of $\mathrm{TB}$ cases to the PHC. Furthermore, the present condition of recording and reporting for tuberculosis cases in the PHC working area was also assessed. The finding of this study can be used to increase PP adherence towards reporting standards and to identify suitable strategies for improving recording and reporting tuberculosis cases at the $\mathrm{PHC}$ level.

\section{Methods}

Subjects of the research

The population targets of this study were all PP in the working area of Babakan Surabaya 
PHC and the head of the PHC. There were 19 PP in the PHC working area. We included PP with valid medical licenses and those who agreed to participate in this research. 15 PP were included as participants. ${ }^{4}$ PP did not agree to participate in this study. The objectives of the research and an anonymous questionnaire form were given orally to the subjects. A face-to-face interview in their workplace was conducted. Those who refused to participate in the study or who have expired medical license were excluded.

\section{Instruments}

The study was conducted with a questionnaire for PP and the head of PHC. The questionnaire for PP consisted of 7 questions to investigate the reasons for PP to report or not report TB cases, 17 questions to characterize the TB program coverage among them (treated cases, treatment outcome, and frequency of reporting). The questionnaire for the head of the PHC was consisted of 22 questions to acquire information about the mechanism of TB recording and reporting in the PHC. Both questionnaires asked 8 questions regarding the necessity of applying the Japanese modification of DOTS components in the future.

TB treatment cards were checked to assess the present condition of recording and reporting at the PHC. A TB treatment card is a record containing a patient's medical information, i.e., identity, treatment history, result of diagnostic tools and sputum smear follow-up, close contact examination (contact tracing), drugs ingestion schedule checklist, treatment outcome, and case classification.

\section{Classification of cases and treatment outcomes}

Treatment outcomes were classified into cured, treatment complete, transferred, and defaulter. A cured case is defined as a case that has been finished completely and has a negative sputum smear follow-up result at the end of the treatment. A treatmentcomplete case is defined as a case that has been finished completely but did not meet the criteria to be classified as cured or as a treatment failure. It applies to sputum smearnegative cases of pulmonary tuberculosis and extrapulmonary cases. The complete cases usually lack a sputum-smear examination result at the beginning or at the end of treatment. A defaulter case is defined as a case in which the patient did not take medication for two months and up. A transferred case is one that was transferred to another health care facilities without knowing the treatment outcome. Ongoing treatment is a case for which the treatment is still being conducted. ${ }^{17}$ The classifications of cases are as follows: sputum smear-positive case of pulmonary tuberculosis, sputum smear-negative case of pulmonary tuberculosis, extrapulmonary, child category, and clinical tuberculosis. Sputum smear-positive case of pulmonary tuberculosis is pulmonary tuberculosis with a positive sputum smear examination result. Sputum smear-negative case of pulmonary tuberculosis is pulmonary tuberculosis with a negative sputum-smear examination result. Extrapulmonary is a tuberculosis case that does not involve lung tissue; it uses a histopathological examination, x-ray, and sputum-smear examination as diagnostic tools. Child category is a tuberculosis case in childhood (0-14 years old), usually diagnosed by the WHO scoring system (including x-ray, tuberculin test, but no need to perform sputum smear examination). Clinical tuberculosis is a case which is diagnosed based on clinical symptoms and $\mathrm{x}$-ray without sputum-smear examination. Sputum-tested cases include sputum smear-positive cases of pulmonary tuberculosis, sputum smearnegative case of pulmonary tuberculosis and extrapulmonary cases. On the other hand, 
non-sputum-tested cases include the clinical tuberculosis cases and child category. ${ }^{18}$

\section{Data analysis}

Collected qualitative data were analyzed by transcription and reduction of respondents' opinions. After coding and categorizing, interpretation of the data was performed. Factors affecting the reporting/non-reporting could be divided into four main reasons (Figure 1): 1) self-awareness, 2) ignorance, 3) lack of time, and 4) poor implementation of recording and reporting at the PHC. The quantitative data were analyzed using nonparametric statistics (Fischer Exact Test) using Easy R (EZR).

\section{Ethical statement}

This study has been approved by the Epidemiologic Research Ethics Committee of Gunma University Faculty of Medicine with no 25-63 in January 2014. Local Government of Bandung City also gave authorization for conducting the study. Verbal consent was obtained from each respondent.

\section{Results and Discussion}

The quantitative analysis of PP opinion regarding the reporting of $\mathrm{TB}$ cases to PHC can be seen in Figure 1. The present condition of recording and reporting in the PHC working area is presented in Figure 2. Total treated cases at the PHC were 42, while those at PP were 52. TB treatment-card usage and monthly follow-up for patient's treatment were performed for all TB cases at the PHC (42 cases). In contrast, card usage and followup were extremely low in the PP sector (1 case). Moreover, there was no contact tracing performed at private practitioners.

Figure 3 shows TB treatment outcomes at the PHC and PP. Cured cases at PP were less than half of those at the PHC. Treatmentcomplete cases at PP were almost one and one-half-fold higher than were those at the PHC. There were three defaulters at PP, while there were none at the PHC. There were four transferred cases at PP, whereas there were none at the PHC. Moreover, there were four cases that were still ongoing at PP and one case at the PHC.

Figure 4 shows the composition of each

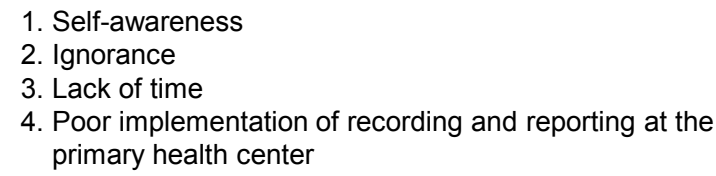

1. Self-awareness

2. Ignorance

3. Lack of time

4. Poor implementation of recording and reporting at the primary health center

Figure 1. Qualitative analysis of private practitioners' opinion 


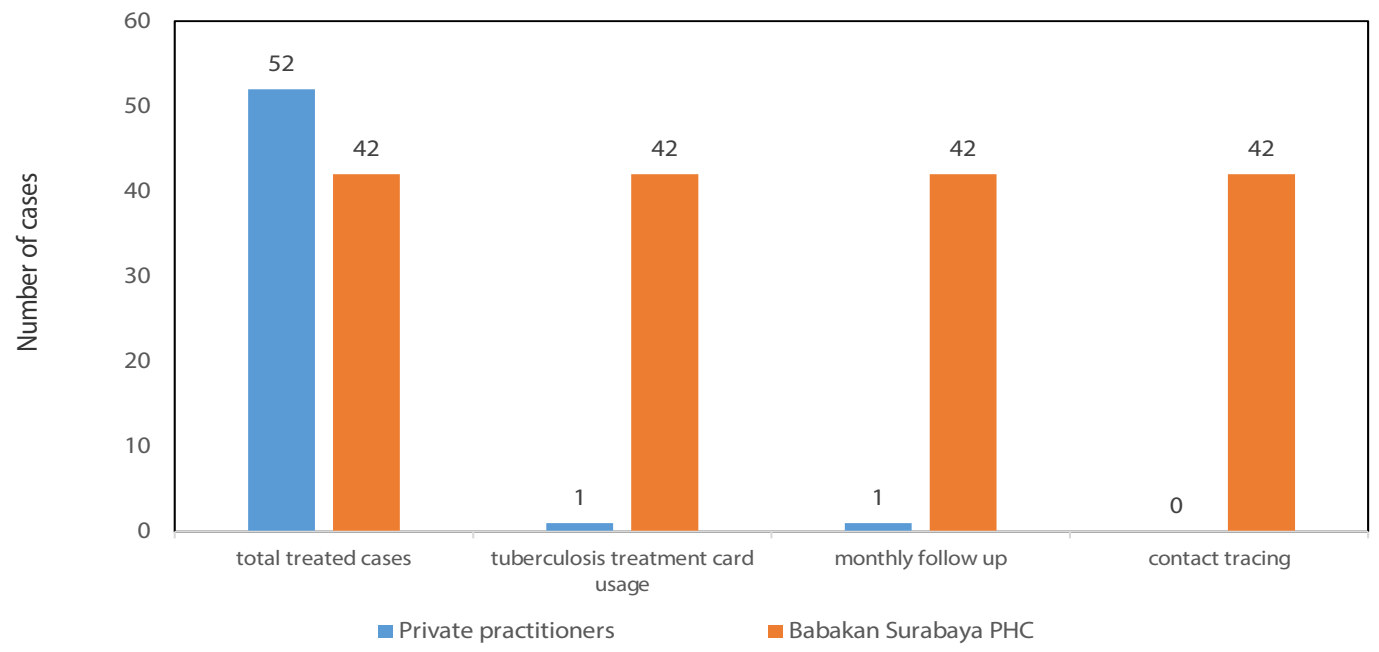

Figure 2. Present condition of tuberculosis recording and reporting at the Babakan Surabaya PHC working area

category of $\mathrm{TB}$ cases at the $\mathrm{PHC}$ and PP. At the PHC, the sputum smear-positive cases of pulmonary tuberculosis $(41 \%)$ represented the largest percentage across all categories, while sputum smear-negative cases of pulmonary TB (31\%) ranked second. Twenty-one percent of TB cases were child category. Extrapulmonary cases represented $5 \%$ across all categories. Clinical TB cases were the fewest across all categories (2\%).
In contrast, clinical $\mathrm{TB}$ had the highest percentage among all categories (38\%) at PP. Meanwhile, sputum smear-positive case of pulmonary TB cases represented only $12 \%$. Child category was considered the smallest part among all categories (4\%). Both sputum smear-negative cases of pulmonary $\mathrm{TB}$ and extra pulmonary cases had the same incidence $(23 \%)$.

Table 2 shows the number of sputum-tested

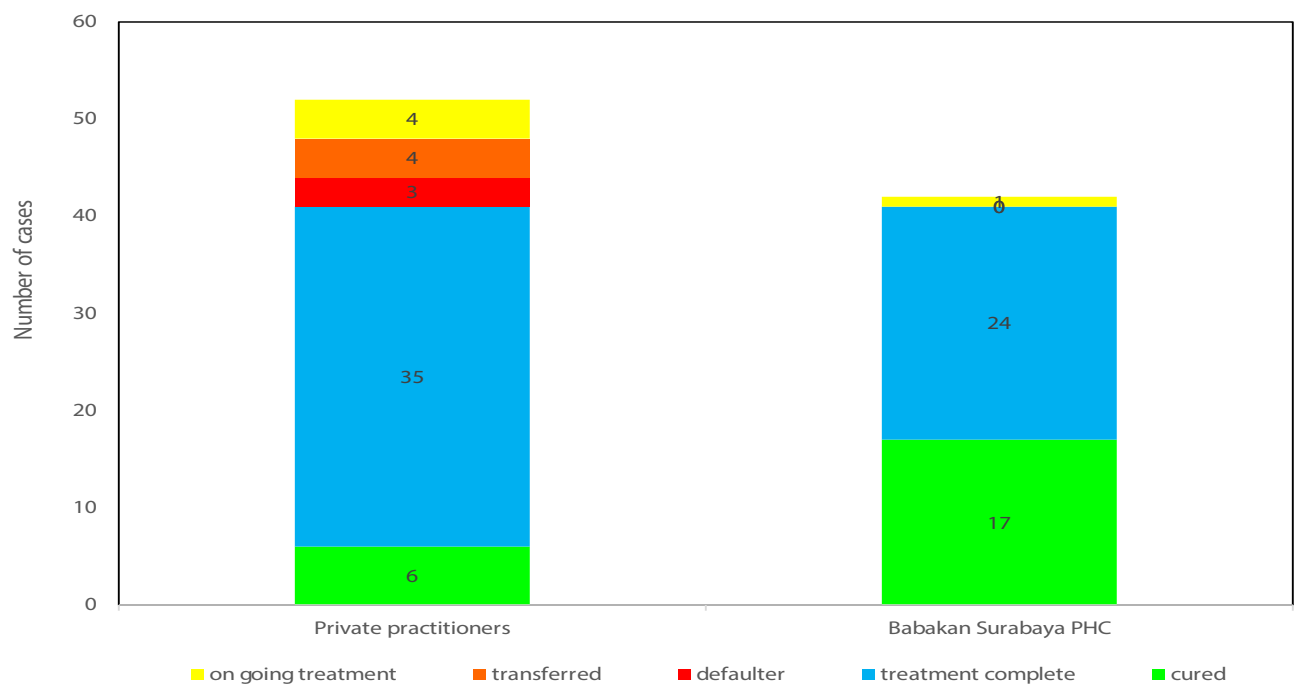

Figure 3. Tuberculosis treatment outcomes at Babakan Surabaya PHC and private practitioners 


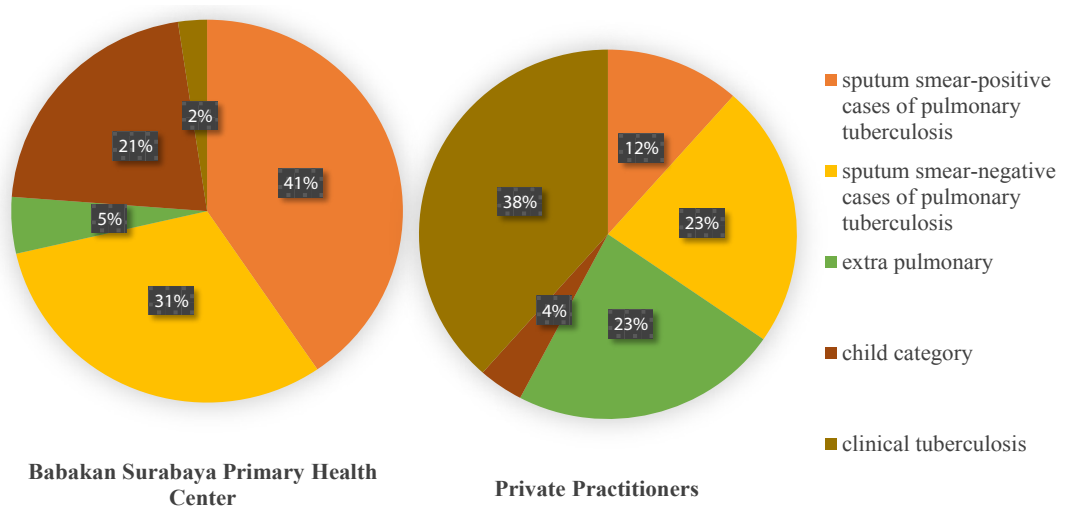

Figure 4. The composition of tuberculosis-treated cases at Babakan Surabaya PHC and private practitioners

and non-sputum-tested TB cases at PP and the PHC. Sputum-tested and non-sputumtested cases at PP were different from those at the PHC $(\mathrm{p}=1.57 \times 10-7)$. There were 30 cases with sputum-smear test at PP and 32 cases at the PHC. On the other hand, there were 22 cases without sputum-smear test at $\mathrm{PP}$ and 10 cases at the PHC. There were more sputum smear-negative cases of pulmonary TB (12 cases) than sputum smear-positive cases of pulmonary TB (6 cases) at PP. In contrast, at the PHC, the number of sputum smear-positive cases of pulmonary TB was higher than sputum smear-negative cases of pulmonary TB. The number of extrapulmonary cases presenting at PP was six times greater than the number presenting at the PHC. Clinical TB cases at PP were twenty times higher than those present at the
PHC.

The level of self-awareness was associated with the PP who reported and did not report TB cases to the center (Table 3) with $p=0.0095$. Two PP had high self-awareness and reported tuberculosis cases. Nine PP had middle self-awareness and did not report the cases. Four PP had low self-awareness and did not report the cases.

Table 4 shows how PP answered each reason described in figure 1. Self-awareness was related to PP's behavior to report TB cases $(\mathrm{p}=0.0095)$. On the other hand, lack of time, ignorance, and poor implementation of recording and reporting at the $\mathrm{PHC}$ were not related to the PP who reported and did not report the cases $(\mathrm{p}=1)$.

Table 1. The coverage of the tuberculosis control program at the PHC in 2012

\begin{tabular}{lcc}
\hline \multicolumn{1}{c}{ Category } & Calculation & Result \\
\hline $\begin{array}{l}\text { Total estimated sputum smear-positive cases of pulmonary } \\
\text { tuberculosis }\end{array}$ & $(107 / 100,000) \times 36,088$ & 38 cases \\
$\begin{array}{l}\text { Number of reported sputum smear-positive cases of pulmo- } \\
\text { nary tuberculosis }\end{array}$ & 14 & 14 cases \\
$\begin{array}{l}\text { Number of estimated unreported sputum smear-positive } \\
\text { cases of pulmonary tuberculosis }\end{array}$ & $38-14$ & 24 cases \\
$\begin{array}{l}\text { Case-finding coverage of sputum smear-positive cases of } \\
\text { pulmonary tuberculosis }\end{array}$ & $14 / 38 \times 100 \%$ & $36 \%$ \\
\hline
\end{tabular}


Table 2. The number of sputum tested and non-tested tuberculosis cases at private practitioners and Babakan Surabaya Primary Health Center (PHC)

\begin{tabular}{l|cc|c|c|c|c}
\hline \multicolumn{4}{c}{ Sputum Tested } & \multicolumn{2}{c|}{ Non-sputum tested } & \multirow{2}{*}{ total } \\
\hline & pulmonary & $\begin{array}{c}\text { extrapulmo- } \\
\text { nary }\end{array}$ & $\begin{array}{c}\text { clinical tuber- } \\
\text { culosis }\end{array}$ & child category & \\
\hline Private practitioners & 6 & 12 & 12 & 20 & 2 & 52 \\
\hline Babakan Surabaya PHC & 17 & 13 & 2 & 1 & 9 & 42 \\
\hline *Fischer Exact test with p-value $=1.57 \times 10-7$
\end{tabular}

*Fischer Exact test with p-value $=1.57 \times 10-7$

Table 5 shows the opinion of PP and the head of the PHC regarding the necessity of adopting the Japanese modification of DOTS components in Bandung. None of the necessity of applying these components was related to the number of PP who reported and did not report cases because all components of the strategy revealed $p>0.05$ by Fischer Exact test. Most PP (up to $70 \%$ respondents) and the head of the PHC claimed that recording and reporting for the follow-up of patients' treatment, private sector involvement, the use of electronic-based reporting, and establishing a time restriction for reporting TB cases were very important strategies to be applied in the future. In contrast, the head of the center and $80 \%$ of the PP implied that active case finding was not necessary in the future. Just $50 \%$ of the PP who reported TB cases agreed that optimizing public health nurses' role as drugs-taking supervisor, conducting cohort meetings regularly with the TB committee and emphasizing preventive activity at the PHC were necessary to be instituted. On the other hand, the head of the PHC considered those strategies not necessary.

Although precise data on TB diagnosis and treatment in the private sector are not available, it is generally believed that about one-third of all TB cases might be managed in the private sector - partly or completely. The previous study, conducted by the Indonesian Medical Association in 2000, showed that each practitioner treated twentyTB patients annually. ${ }^{19}$ Our findings showed that the number of TB cases treated at PP was higher than was the number treated at the PHC. Unfortunately, just two respondents reported cases regularly to the PHC. Therefore, there were many cases not reported to the PHC. However, if they were well recorded and reported, it will help the $\mathrm{PHC}$ to improve TB prevention, diagnosis and treatment services. If contact tracing, as one of the prevention activities, could be well implemented, drug resistance and further spreading of TB could be reduced. ${ }^{20,21}$

A TB treatment card was used for only one case at PP (figure 2). Therefore, there were

Table 3. Level of self-awareness that makes private practitioners report or not report tuberculosis cases to the primary health center

\begin{tabular}{lcccc}
\hline \multirow{2}{*}{ Private practitioners } & \multicolumn{3}{c}{ Self-awareness } & \multirow{2}{*}{ Total } \\
\cline { 2 - 4 } & High & Middle & Low & \\
\hline Reported & 2 & 0 & 0 & 2 \\
Not reported & 0 & 9 & 4 & 13 \\
Total & 2 & 9 & 4 & 15 \\
\hline
\end{tabular}

*Fischer Exact test with p-value $=0.0095$ 
Table 4. Reasons that influence PPs to report or not to report tuberculosis cases to the PHC

\begin{tabular}{|c|c|c|c|c|c|c|}
\hline \multirow[t]{2}{*}{ Reason } & \multicolumn{2}{|c|}{$\begin{array}{l}\text { Number of PPs who } \\
\text { report and answer }\end{array}$} & \multicolumn{2}{|c|}{$\begin{array}{c}\text { Number of PPs who } \\
\text { do not report and } \\
\text { answer }\end{array}$} & \multirow[t]{2}{*}{ TOTAL } & \multirow[t]{2}{*}{$*_{\mathrm{p}}$-value } \\
\hline & yes & no & yes & no & & \\
\hline Self-awareness & 2 & 0 & 0 & 13 & 15 & 0.0095 \\
\hline Ignorance & 0 & 2 & 4 & 9 & 15 & 1 \\
\hline Lack of time & 0 & 2 & 3 & 10 & 15 & 1 \\
\hline $\begin{array}{l}\text { Poor implementation of re- } \\
\text { cording and reporting at the } \\
\text { primary health center }\end{array}$ & 0 & 2 & 2 & 11 & 15 & 1 \\
\hline
\end{tabular}

only a few cases of patients' treatment monthly follow-up and contact tracing. Without using a TB treatment card, it is difficult for physicians to perform those activities. Several PP said that completing the TB treatment card was too complicated and required more time. They therefore did not have time to use it. This situation indicates that recording and reporting at PP did not function well.

There were a small number of cured cases and a high number of treatment-complete cases at PP (Figure 3). The fact that there were many cases without a sputum-smear examination result at the end of treatment might increase the spreading of TB. The defaulters occurring at PP were probably caused by low use of the PP treatment card. Due to this situation, monthly patients' treatment follow-up was conducted only rarely at PP. Accordingly, it can be summarized that recording and reporting for TB did not function comprehensively at PP. The difference between number of sputum tested and number of non-sputum tested at PP and the PHC (table 2) was due to low participation of PP in DOTS activities. Accordingly, low numbers of sputum-smear examinations appeared primarily at the PP. Several studies also showed that clinicians, in particular those who work in the private sector, often deviate from standard, internationally recommended tuberculosis management practice. These deviations include underutilization of the sputum-smear examination, over-reliance on radiography for diagnosis, and failure to supervise and assure adherence to treatment. ${ }^{22,23}$

Several PP reported regularly, as they realized that they had to do it. They thought that recording and reporting was very important for reducing TB cases in the community. Thus, it can be assumed that they had selfawareness. On the other hand, some of them argued that recording and reporting was not their responsibility. They considered it was not important and wasted their time. They tended to have low self-awareness (table 3 ). However, some of them did not know that they had an obligation to report the cases regularly to the authorized PHC (table 4). A few PP focused on treating patients only, so that they usually did not have time to report (table 4). These results indicate that disseminating recent information about TB recording and reporting was important for reducing the PP ignorance and improving their level of self-awareness. 
Poor implementation of recording and reporting at the center, such as incomplete feedback for private practitioners who already reported cases and no active report collecting mechanism, made them fail to report (Table 4). Good communication may offer a solution for this situation because it can bridge private practitioners' needs and the requirements of the center. Moreover, it can motivate private practitioners to report, as they appreciated being asked their opinions regarding how to solve the problems collaboratively.

Several strategies may improve the adherence of PP towards recording and reporting standards in TB cases. The strategy should focus particularly on increasing the level of self-awareness. However, lack of coordination and communication between the primary health center and private practitioners will lead to private practitioners' ignorance in reporting tuberculosis cases to the center. Therefore, private sector involvement in DOTS activities should be improved. A monthly meeting, electronic-based reporting could be employed to enhance the selfawareness of the PP. ${ }^{24,25}$

\section{Conclusions}

The level of self-awareness influenced PP's behavior regarding TB case reporting to the PHC. Construction of a wellestablished recording and reporting system for tuberculosis cases in Bandung requires private-sector involvement, improvement of recording and reporting for patients' treatment follow-up, and implementation and use of electronic-based reporting. Additionally, good communication and collaboration between the primary health center and private practitioners are needed. This can be achieved by strengthening the public-private mix system. Moreover, regular meetings are recommended to support coordination and partnership between private practitioners and the primary health center.

\section{Acknowledgement}

We would like to thank the Faculty of Medicine at Padjadjaran University and the Department of Public Health Graduate School of Medicine at Gunma University for their help and advice in conducting this study.

\section{Funding}

The study was not funded by any source of grants.

\section{Conflict of Interest}

The authors declared no potential conflicts of interest with respect to the research, authorship, and/or publication of this article.

\section{References}

1. Lobue PA, Enarson DA. Tuberculosis in humans and its epidemiology, diagnosis and treatment in the United States. International Journal of Tuberculosis and Lung Diseases. 2010; 14:1226-32.

2. Sandhu GK. Tuberculosis: current situation, challenges and overview of its control programs in India. Journal of Global Infectious Diseases. 2011;3(2):143-150.

3. Lönnroth K, Raviglione M. The WHO's TB Strategy in the era of the Sustainable Development Goals. Transactions of the Royal Society of Tropical Medicine and Hygiene. 2016;110(3):148-150.

4. Glaziou P, Sismanidis C, Floyd K, Global epidemiology of tuberculosis. Cold Spring Harbor Perspectives in Medicine. 2015;5(2):a017798.

5. Sulis G, Roggi A, Matteelli A, Raviglione MC. Tuberculosis: Epidemiology and Control. Mediterranean Journal of Hematology and Infectious Diseases. 2014;6(1):e2014070.

6. Tiemersma EW, van der Werf MJ, Borgdorff MW, Williams BG, Nagelkerke 
NJD. Natural history of tuberculosis: duration and fatality of untreated pulmonary tuberculosis. PLoS ONE. 2011;6(4):e17601.

7. Raviglione M, Sulis G. Tuberculosis 2015: Burden, Challenges and Strategy for Control and Elimination. Infectious Disease Reports. 2016;8(2):6570.

8. Azhar GS. DOTS for TB relapse in India: A systematic review. Lung India. 2012;29(2):147-153.

9. Karumbi J, Garner P. Directly observed therapy for treating tuberculosis. The Cochrane Database of Systematic Reviews. 2015;(5):1-56.

10. Ahmad RA, Mahendradhata Y, Cunningham J. How to optimize tuberculosis case finding: explorations for Indonesia with a health system model. BMC Infectious Diseases. 2009;9:87.

11. Nair N, Wares F. Tuberculosis in the SouthEast Asia region. Bulletin of the World Health Organization. 2010;88(3):164.

12. Billingsley KM, Smith N, Shirley R, Achieng L, Keiser P. A quality assessment tool for tuberculosis control activities in resource limited settings. Tuberculosis. 2011;91S1:S49-S53.

13. Katsuda N, Hirosawa T, Reyer JA, Hamajima N. Roles of public health centers (Hokenjo) in tuberculosis control in Japan. Nagoya Journal of Medical Science. 2015;77(1-2):19-28.

14. Bandung District Health Office. Bandung City Health Profile 2012. Bandung, West Java, Indonesia.

15. Lai PC, Low CT, Tse WS. Risk of tuberculosis in high-rise and high density dwellings. Environment and Pollution. 2013;183:40-45.

16. Babakan Surabaya Primary Health Center. Annual primary health care report 2012. Bandung, West Java, Indonesia.

17. Bhavnani D, Lancki N, Winter I, Macaraig M. Treatment outcomes of patients with tuberculosis in New York City. Journal of
Public Health Management and Practice. 2015. 21(6):11-8.

18. Horne DJ, Royce SE, Gooze L. Sputum monitoring during tuberculosis treatment for predicting outcome. The Lancet Infectious Diseases. 2010;10(6):387-394.

19. Naseer M, Khawaja A, Pethani AS. How well can physicians manage Tuberculosis. BMC Health Services Research. 2013; 13:439.

20. World Health Organization. Public Private-Mixed DOTS in Indonesia: A Strategy for Action. Geneva: 2003.

21. Hazarika I. Role of Private Sector in providing tuberculosis care: evidence from a population-based survey in India. Journal of Global Infectious Diseases. 2011;3(1):19-24.

22. Chengsorn N, Bloss E, Anekvorapong R, Anuwatnonthakate A, Wattanaamornkiat W, Komsakorn S, et al. Tuberculosis services and treatment outcomes in private and public health care facilities in Thailand, 2004-2006. International Journal of Tuberculosis and Lung Disease. 2009; 13:888-894.

23. Tauro CK, Gawde NC. Diagnosis and treatment of childhood pulmonary tuberculosis: a cross-sectional study of practices among paediatricians in private sector. Interdisciplinary Perspectives on Infectious Diseases. 2015;2015:960131.

24. Huang F, Cheng S, Du X. Electronic recording and reporting system for tuberculosis in China: experience and opportunities. Journal of the American Medical Informatics Association: JAMIA. 2014;21(5):938-941.

25. Sharma A, Ndisha M, Ngari F. A review of data quality of an electronic tuberculosis surveillance system for case-based reporting in Kenya. European Journal of Public Health. 2015;25(6):1095-1097. 